Michigan Health System. Suitability for mitralvalve repair was related to age, ejection fraction and left-ventricular dimension. Of 419 patients for whom surgery was deemed appropriate, 126 actually underwent the procedure. No significant difference in survival rates was observed between candidates who underwent surgery and those who were treated medically. In addition, the combined endpoint of death, implantation of a left-ventricular assist device, or heart transplantation occurred in a similar proportion of patients in both groups.

Concluding that mitral valve annuloplasty conferred no survival advantage in this cohort of patients, the authors recommend that a prospective, randomized study should be carried out to determine which patients are likely to benefit from the procedure.

Original article Wu AH et al. (2005) Impact of mitral valve annuloplasty on mortality risk in patients with mitral regurgitation and left ventricular systolic dysfunction. J Am Coll Cardiol 45: 381-387

\section{Mitral regurgitation and myocardial infarction}

There is evidence that mitral regurgitation increases the risk of death following myocardial infarction. A recent study by Bursi and colleagues has investigated the associa- tion between mitral regurgitation, heart failure and death in a cohort of myocardial infarction survivors in Minnesota.

Of 1,331 patients experiencing their first myocardial infarction, 773 underwent echocardiography within 30 days of the event. Semiquantitative assessment by color Doppler showed that mitral regurgitation was present in half of these patients. The condition was mild in most patients, although a quarter of cases were moderate or severe. During a follow-up of almost 5 years, patients with mitral regurgitation had worse outcomes, irrespective of age, sex, left-ventricular ejection fraction or Killip class. Those with moderate or severe mitral regurgitation were more than three times more likely to suffer heart failure than those without the condition, and their risk of death beyond 30 days was increased by approximately a half. The prevalence of mitral regurgitation was not associated with the location of the infarct.

Bursi et al. conclude that mitral regurgitation is common among patients with myocardial infarction. Since the condition is often unrecognized and appears to increase the risk of heart failure and death in these patients, they propose that it should be routinely assessed during postmyocardial infarction risk stratification.

Original article Bursi L et al. (2005) Heart failure and death after myocardial infarction in the community: the emerging role of mitral regurgitation. Circulation 111:295-301 